\title{
纳米电极上单个银纳米颗粒氧化电流分辨能力的研究
}

\author{
杨哲曜马巍应佚伦龙亿涛* \\ (华东理工大学 化学与分子工程学院 结构可控先进功能材料及其制备教育部重点实验室 上海 200237)
}

\begin{abstract}
摘要 在单体电化学的研究中, 提高信号分辨能力是一项挑战. 缩小电极尺寸有利于对体系噪音电流的控制, 有望提 高电流的分辨能力. 本研究制备了直径为 $480 \mathrm{~nm}$ 的铂纳米圆盘电极, 选用银纳米颗粒碰撞电极产生银电化学氧化行为 作为模型, 考察了纳米电极相对于微米电极在单体电化学信号分辨能力上的优化作用. 研究表明, 不同尺寸电极上观 察到的银纳米颗粒的碰撞频率符合扩散控制的碰撞规律. 说明单个电流信号对应于单个纳米颗粒的电化学氧化过程. 同时, 当电极尺寸缩小至纳米尺度后，噪音电流下降 $50 \%$ 左右，提高了对银纳米颗粒碰撞电极过程中氧化电流的分辨 能力. 研究结果表明使用纳米电极能进一步提高对单体电化学中微小电流的检测能力.
\end{abstract}

关键词 单颗粒电化学; 纳米电极; 碰撞频率; 高电流分辨

\section{Study on the Resolution of Single Silver Nanoparticles Electrochemical Behavior at Nanoelectrode}

\author{
Yang, Zheyao Ma, Wei Ying, Yilun Long, Yitao* \\ (Key Laboratory for Advanced Materials, School of Chemistry \& Molecular Engineering, \\ East China University of Science and Technology, Shanghai 200237)
}

\begin{abstract}
Single entity electrochemistry (SEC) has been attracting increasing interests over the past few years because of its extremely high sensitivity. This method offers the penetrating insights into the properties of individual entities that are masked in traditional ensemble measurements. Electrocatalytic amplification, blocking and direct electrochemical reaction of individual entities by detecting the current transients were employed as single entity collides at an electrode. However, it remains a challenge to enhance the current resolution in the SEC field, especially for the complex electrochemical behaviors. In this work, a strategy using a small-sized ultramicroelectrode and nanoelectrode was performed to reduce both background current and collision frequency, which allowed to reach the typical electrochemical signals. A low-noise electrochemical measurement system was used to acquire the data of single silver nanoparticles (AgNPs) collision at $480 \mathrm{~nm}$ Pt nanoelectrode and $10 \mu \mathrm{m}$ ultramicroelectrode. The electrochemical measurement was carried out in $20 \mathrm{mmol} \cdot \mathrm{L}^{-1}$ phosphate buffer $(\mathrm{pH}=$ 7.4) at an applied potential of $+0.6 \mathrm{~V}$ vs. $\mathrm{Ag} / \mathrm{AgCl}$ wire in the presence of $58 \mathrm{~nm} \mathrm{AgNPs}$. The sampling rate was of $100 \mathrm{kHz}$ by using an A/D convertor and the low-pass fitter was set at $5 \mathrm{kHz}$. Signal-noise ratio was improved by $50 \%$ when the diameter of working electrode decreased from $10 \mu \mathrm{m}$ to $480 \mathrm{~nm}$, resulting in more detailed information available at nanoelectrode during the collision processes of individual AgNPs. Both the employed nanoelectrode as working electrode and low-noise electrochemical measurement platform can significantly enhance the current resolution of SEC. High current resolution signals with picoampere and sub-millisecond sensitivity were observed for electrochemical oxidation of single AgNPs on nanoelectrode. In addition, the experimentally observed collision frequencies at varying size of ultramicroelectrode and nanoelectrode were in reasonable agreement with the theoretically calculated ones by Fick's Diffusion Laws within a typical variation associated with stochastic measurements. The electrochemical result indicate that individual AgNPs collisions are governed mainly by diffusion process. The high accuracy of the proposed current signal makes it possible to understand the electrochemical behavior of individual AgNPs as a function of the dwell time. Our results have demonstrated that the nanoelectrode would be a powerful platform for better delivering a complete picture of electrochemical behavior of individual entities, visualization of the electrons transfer process at single entity level.

Keywords single nanoparticle electrochemistry; nanoelectrode; collision frequency; high current resolution
\end{abstract}

\section{1 引言}

单个实体电化学(简称单体电化学, Single entity electrochemistry)包括对单个纳米材料(纳米颗粒、纳米
管及纳米线)的电化学和电催化活性、单个功能材料的 性能、单个生物分子行为等进行灵敏检测的电化学技 术 $^{[1]}$. 单体电化学技术在电极上获得单个实体的电化学 信号 ${ }^{[2,3]}$, 从而提供了一种新手段使得从局部到整体地

*E-mail: ytlong@ecust.edu.cn

Received March 29, 2017; published May 8, 2017.

Project supported by the National Natural Science Foundation of China (Nos. 21421004, 21327807) and Fundamental Research Funds for the Central Universities (No. 222201313004).

项目受国家自然科学基金(Nos. 21421004, 21327807)和中央高校基本科研业务费专项资金(No. 222201313004)资助. 
对电化学进行理解成为可能. 目前, 利用该技术已实现 了对单个纳米颗粒 ${ }^{[4,5]}$ 、单个乳滴 ${ }^{[6]}$ 、单个囊泡 ${ }^{[7]}$ 和单个 病毒 ${ }^{[8]}$ 等单体的分析检测. 其中, 以单个银纳米颗粒作 为模型的电化学氧化过程的研究较为系统. 最近的研究 表明, 单个银纳米颗粒的电化学氧化行为与纳米颗粒的 运动轨迹相关, 其电流信号包含一些因电流值低而较难 检测的细节信息 ${ }^{[9 \sim 11]}$. 因此, 对单个银纳米颗粒碰撞电 极时电化学氧化信号的精确测量就显得尤为重要. 电极 尺寸的缩小有利于对体系噪音电流的控制 ${ }^{[12]}$. 因此, 我 们利用纳米电极的优势提高电流的分辨率, 进而对碰撞 过程时的电化学信号实现精确测量. 然而现有的报道 中, 利用纳米电极进行电化学碰撞的研究相对较少, 这 与纳米电极制备复杂、重复性难以保证、极易损坏等因 素相关 ${ }^{[13,14]}$, 而由纳米电极带来的动力学变化是否会对 银纳米颗粒氧化行为的精确测量产生影响也尚属未知. 此外, 理论上电极尺寸越小噪音电流相应也越小, 但是 仪器噪音的存在使得更小尺寸的电极在电流噪音的控 制上存在瓶颈. 因此, 本研究选用直径为 $480 \mathrm{~nm}$ 的铂圆 盘纳米电极, 考察其在银纳米颗粒电化学碰撞实验中的 信号分辨能力. 通过使用低噪音、高带宽、高采样频率 的皮安级微电流放大器对电流信号进行记录. 对不同尺 寸电极在银纳米颗粒碰撞电极过程中记录电流信号的 分析表明, 直径为 $480 \mathrm{~nm}$ 铂圆盘电极的噪音电流比 10 $\mu \mathrm{m}$ 铂圆盘电极的噪音电流降低 50\%左右, 小尺寸电极 对单个银纳米颗粒碰撞电极产生的氧化电流具有更高 的分辨能力, 从而实现了对单个银颗粒电化学氧化信号 的精确测量. 此外, 不同尺寸的铂圆盘电极上发生的银 纳米颗粒碰撞频率与单颗粒扩散控制的理论碰撞频率 相匹配, 证明了单个电流信号对应于单个纳米颗粒的碰 撞. 因此, 使用纳米电极可以提高对微弱电流的检测能 力, 有利于实现单体电化学的准确测量.

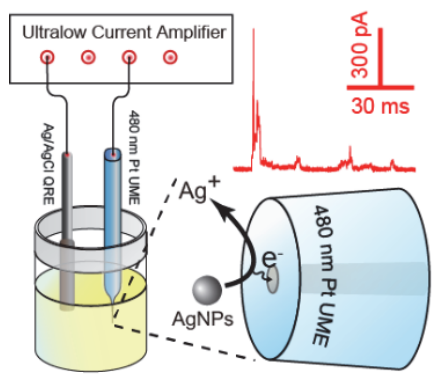

图 1 铂超微电极上银纳米颗粒电化学碰撞示意图, 电极直径为 10 $\mu \mathrm{m}$ 或 $480 \mathrm{~nm}$

Figure 1 Schematic depiction of AgNPs collision at $10 \mu \mathrm{m}$ or $480 \mathrm{~nm}$ Pt UME

\section{2 结果与讨论}

\section{1 微/纳电极及银纳米颗粒的表征}

本研究制备的直径为 $480 \mathrm{~nm}$ 的纳米电极, 通过稳 态电流、扫描电子显微镜(SEM) 和光学图像分别对电极 尺寸、形貌及电化学性能进行表征. 图 2 示出微/纳铂圆
盘电极在 $5 \mathrm{mmol} \cdot \mathrm{L}^{-1}$ 二茂铁 $(\mathrm{Fc})$ 溶液中的循环伏安曲 线. 图示的纳米电极的循环伏安曲线由原始电流放大 5 倍作图得到. 从图中可见，微米电极的稳态电流为放大 后纳米电极稳态电流的 4 倍. 对于圆盘超微电极, 有半 无限球形扩散控制的稳态电流公式:

$$
i_{\mathrm{SS}}=4 n F D C a
$$

式中 $n$ 为电子转移数目, $F$ 为法拉第常数, $D$ 和 $C$ 分别为 二茂铁的扩散系数 $\left(2.45 \times 10^{-9} \mathrm{~m}^{2} \cdot \mathrm{s}^{-1}\right)$ 和浓度 $(5 \mathrm{mmol} \bullet$ $\left.\mathrm{L}^{-1}\right), a$ 为电极半径. 据此, 可以计算得到微米电极的直 径为 $10.2 \mu \mathrm{m}$, 而纳米电极的直径约为 $510 \mathrm{~nm}$. 内插图 $\mathrm{a}$ 为纳米电极的 SEM 图, 示出其直径约为 $480 \mathrm{~nm}$. 内插 图 $\mathrm{b}$ 为微米电极的光学图像, 示出其直径约为 $10.0 \mu \mathrm{m}$.

利用透射电子显微镜(TEM)对银纳米颗粒进行表 征, 结果如图 3 所示. 可见银纳米颗粒的粒径分布均一, 且几何形貌近似于球形. 进一步使用动态光散射对颗粒 粒径进行验证，结果显示其粒径分布于 $58 \mathrm{~nm}$ 附近(内 插图).

\section{2 微/纳电极上银纳米颗粒氧化信号分辨}

为了对微/纳电极的电流分辨能力进行研究, 选用 银纳米颗粒碰撞电极产生的电化学行为作为模型, 分别 进行对比. 将 $480 \mathrm{~nm}$ 和 $10 \mu \mathrm{m}$ 电极分别置于 $20 \mathrm{mmol}$ $\mathrm{L}^{-1}$ 磷酸缓冲液内, 施加 $+0.6 \mathrm{~V}$ 的电位 (双电极系统, 参 比电极/对电极为 $\mathrm{Ag} / \mathrm{AgCl}$ 丝). 溶液中未加入银纳米颗 粒时电流-时间曲线都是光滑、无尖刺的背景电流信号. 而后，将银纳米颗粒加入溶液，可以观察到明显的尖刺 信号(图 $4 \mathrm{~A}-\mathrm{a} 、 \mathrm{~B}-\mathrm{a})$. 上述尖刺信号归因于单个银纳米颗

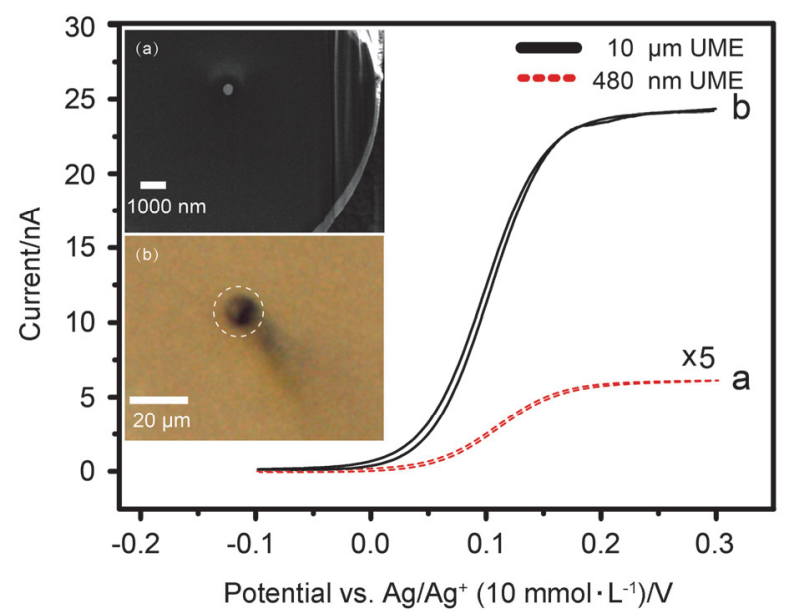

图 2 直径为 $10 \mu \mathrm{m}$ 和 $480 \mathrm{~nm}$ (循环伏安图中纳米电极的电流值五倍 于原始值以方便展示)的铂微/纳电极在 $5 \mathrm{mmol} \cdot \mathrm{L}^{-1}$ 二茂铁和 0.2 $\mathrm{mol} \cdot \mathrm{L}^{-1}$ 四丁基六氟磷酸胺乙腈溶液中的循环伏安曲线, 参比电极为 $\mathrm{Ag} / \mathrm{Ag}^{+}$电极(内参比溶液为 $10 \mathrm{mmol} \cdot \mathrm{L}^{-1} \mathrm{AgNO}_{3}$ 乙腈溶液), 扫速为 50 $\mathrm{mV} \cdot \mathrm{s}^{-1}$. 内插图 (a) 为纳米电极的扫描电镜图, 内插图(b)为微米电极 的光学显微镜图

Figure 2 Cyclic voltammogram of $10 \mu \mathrm{m}$ and $480 \mathrm{~nm}$ (The current is 5 times enlarged in the plot) $\mathrm{Pt} \mathrm{UME}$ in $5 \mathrm{mmol} \cdot \mathrm{L}^{-1} \mathrm{Fc}$ solution, containing $0.2 \mathrm{~mol} \cdot \mathrm{L}^{-1} \mathrm{TBAPF}_{6}$, reference electrode is $\mathrm{Ag} / \mathrm{Ag}^{+}\left(10 \mathrm{mmol} \cdot \mathrm{L}^{-1} \mathrm{Ag}\right.$ $\mathrm{NO}_{3}$ ), at a scan rate of $50 \mathrm{mV} \cdot \mathrm{s}^{-1}$. Insert figure (a) is the SEM image of the nanoelectrode, (b) is the image of microelectrode 


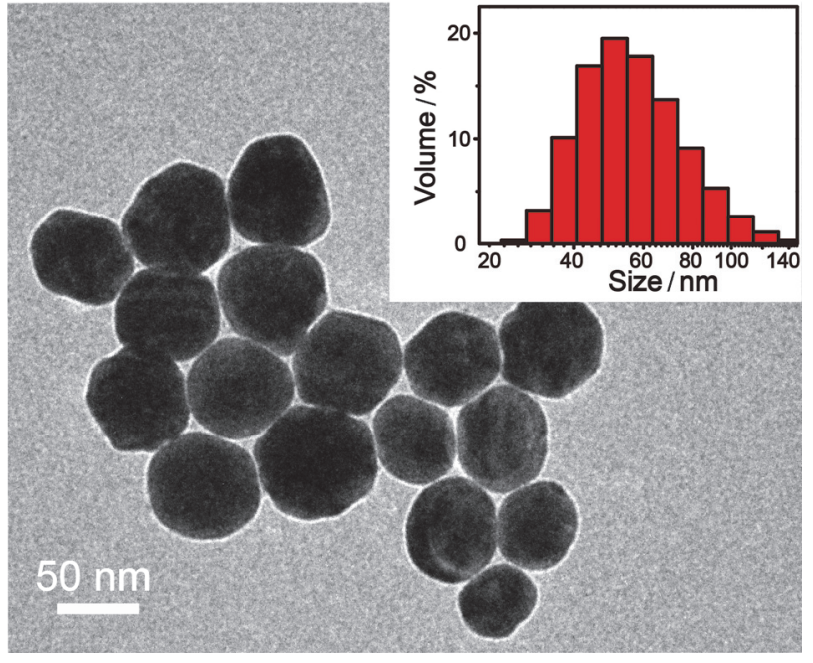

图 3 银纳米颗粒的透射电子显微镜图, 内插图为银纳米溶胶的粒径 分布图

Figure 3 TEM image of silver nanoparticles. Insert figure shows the dynamic light scattering result

粒在电极上的氧化过程 ${ }^{[15,16]}$. 对比碰撞过程中两种电极 的电化学行为差异, 可以观察到在 $480 \mathrm{~nm}$ 的铂圆盘电 极上发生的银纳米颗粒的碰撞频率明显小于在 $10 \mu \mathrm{m}$ 的铂圆盘电极上发生的银颗粒碰撞频率. 在小尺寸电极 上观察到的碰撞频率的降低是由于电极面积降低, 纳米 颗粒碰撞电极的概率降低造成的. 单个纳米颗粒的电化 学碰撞频率受纳米颗粒在溶液中的扩散控制时, 其碰撞 频率 $f$ 满足式 $(2)^{[17,18]}$ :

$$
f=4 a C_{\mathrm{n}} D_{\mathrm{n}} N_{\mathrm{A}}
$$

式中 $C_{\mathrm{n}}$ 为纳米颗粒的浓度, $D_{\mathrm{n}}$ 为纳米颗粒的扩散系数,
$N_{\mathrm{A}}$ 为阿伏伽德罗常数. 其中 $D_{\mathrm{n}}$ 可以根据 Stokes-Einstein 方程进行估算:

$$
D_{\mathrm{n}}=k_{\mathrm{b}} T / 6 \pi \eta r_{\mathrm{n}}
$$

式中 $k_{\mathrm{b}}$ 是 Boltzmann 常数, $T$ 为温度 $(293 \mathrm{~K}), \eta$ 为溶液粘 度 $\left(1.005 \times 10^{-3} \mathrm{~Pa} \bullet \mathrm{s}\right), r_{\mathrm{n}}$ 为颗粒半径 $(29 \mathrm{~nm})$. 式(2)成立 的前提是银纳米颗粒在溶液中的运动方式仅为自由扩 散. 若本研究中纳米颗粒发生聚集, 势必会造成扩散的 抑制和纳米颗粒浓度的降低，从而使得实验观察到的碰 撞频率小于理论计算值. 因此可以根据电流信号频率研 究图 4 中的电化学信号是否为单颗粒行为. 根据式(2)和 式(3)进行理论计算得到 $480 \mathrm{~nm}$ Pt UME 上的碰撞频率 为 $0.074 \mathrm{~s}^{-1}$. 实验中, 根据加入银纳米颗粒后 $200 \mathrm{~s}$ 内观 察到的平均碰撞频率为 $0.064 \mathrm{~s}^{-1}$. 理论计算值和实验观 察值相匹配说明单个电流信号对应于单个银纳米颗粒 的碰撞行为. 图 $4 \mathrm{~A}-\mathrm{a} 、 \mathrm{~B}-\mathrm{a}$ 的内插图为不同尺寸电极在 银纳米颗粒电化学氧化过程中采集的电流基线的放大. 从图中可见, 使用 $10 \mu \mathrm{m}$ 铂圆盘电极作为工作电极采集 的噪音电流较大, 其均方根(RMS)约为 $5.9 \mathrm{pA}$. 而相同 条件下 $480 \mathrm{~nm}$ 铂圆盘电极采集的噪音电流的 RMS 值仅 为 $2.7 \mathrm{pA}$, 相较于微米电极降低了 $50 \%$ 左右. 由于选用 $5 \mathrm{kHz}$ 的滤波器截止滤波, 本研究在较低电流噪音的基 础上, 仍具有强的时间分辨能力 ${ }^{[9]}$.

对比图 4 A-a 及 B-a 中不同大小的电极产生的电流 信号，峰电流未出现明显下降，说明电极面积的减少并 未影响银纳米颗粒的法拉第电子转移过程. 图 4 A-b、 B-b 分别对应于图 4 A-a、B-a 相应标记信号的放大，可 见在 $480 \mathrm{~nm}$ Pt UME 和 $10 \mu \mathrm{m}$ Pt UME 上均可观察到由 一系列独立电流峰构成的多峰电流信号，此现象归因于
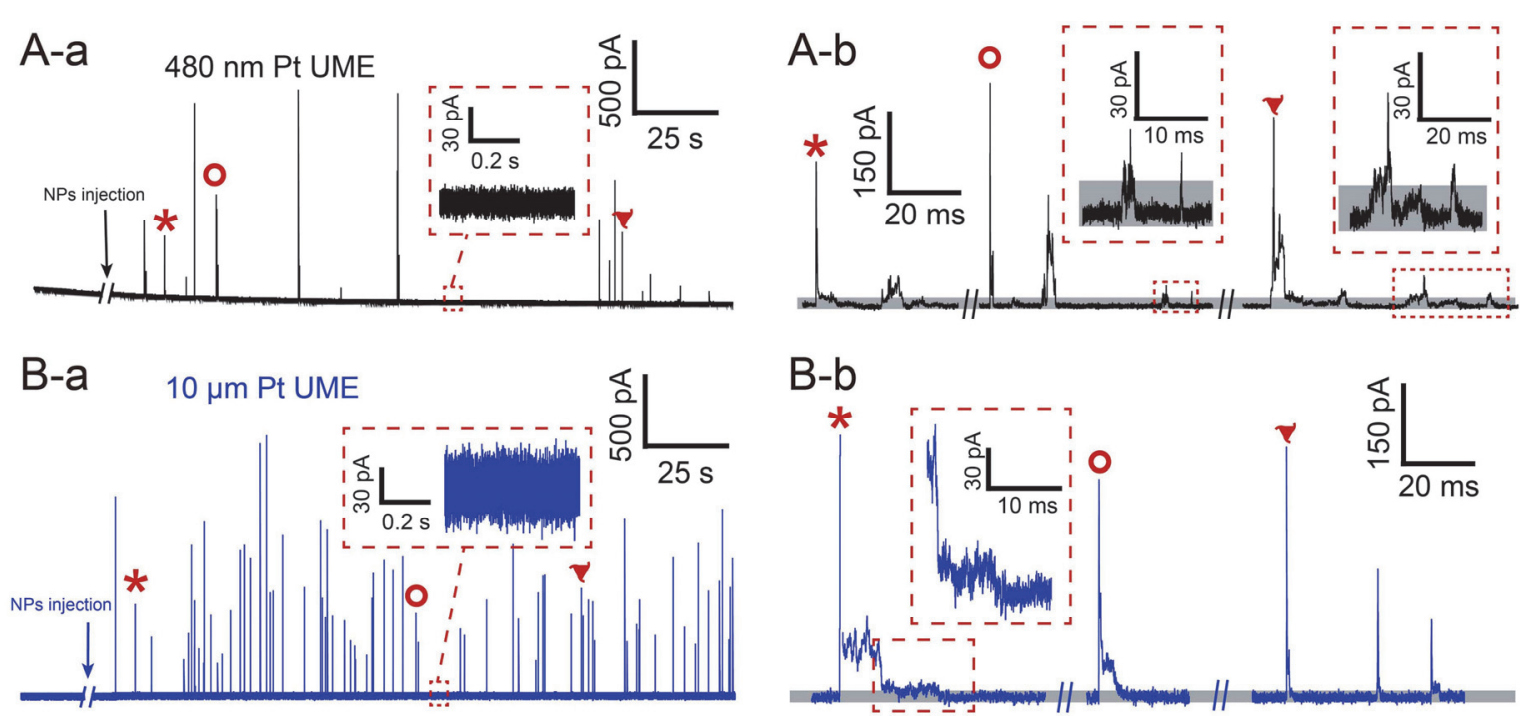

图 4 A-a 和 B-a 分别是 $480 \mathrm{~nm}$ 和 $10 \mu \mathrm{m}$ 铂圆盘电极在加入银纳米颗粒前及加入后的电流响应图. A-b 和 B-b 分别是 $480 \mathrm{~nm}$ 和 $10 \mu \mathrm{m}$ 铂圆盘电 极上单个银纳米颗粒碰撞的电流信号放大图. 内插图为选定区域的放大. 电解质为 $20 \mathrm{mmol} \cdot \mathrm{L}^{-1}$ 磷酸盐缓冲液 $(\mathrm{pH}=7.4)$, 施加电位为 $+0.6 \mathrm{~V} \mathrm{vs}$. $\mathrm{Ag} / \mathrm{AgCl} \mathrm{QRE}$

Figure 4 A-a and B-a are the current response of $10 \mu \mathrm{m}$ and $480 \mathrm{~nm}$ Pt UME in the absence/presence of AgNPs; insert figures are the enlargement of baseline. A-b and B-b are the enlargement of the signals; insert figures are the enlargement of detail information. The experiment was performed in 20 $\mathrm{mmol} \cdot \mathrm{L}^{-1}$ phosphate buffer $(\mathrm{pH}=7.4)$ at applied potential of $+0.6 \mathrm{~V} \mathrm{vs.} \mathrm{Ag} / \mathrm{AgCl} \mathrm{QRE}$ 
银纳米颗粒在碰撞过程中多次碰撞的运动轨迹 ${ }^{[9 \sim 11]}$. 图 4 B-a 及 B-b 中灰色背景的高度对应 $10 \mu \mathrm{m}$ Pt UME 的噪 音大小, 可见在 $480 \mathrm{~nm}$ Pt UME 上产生银纳米颗粒的氧 化信号具有更低的噪音电流, 在直径为 $480 \mathrm{~nm}$ 的电极 上产生的电流信号中, 可以观察到有一部分电流值较低 的信号峰高度低于灰色背景的高度. 显然, 作为工作电 极, 直径为 $480 \mathrm{~nm}$ Pt UME 对单个银纳米颗粒碰撞电极 产生的银纳米颗粒的氧化电流分辨率更高, 可以对单个 银颗粒电化学氧化的信号进行更精确的测量.

\section{3 结论}

本研究分别考察了微/纳尺寸电极在银纳米颗粒氧 化过程中的电化学信号分辨能力. 实验结果表明, 不同 尺寸电极的碰撞频率受颗粒扩散控制, 进而可以得知单 个电流信号对应于单个纳米颗粒的碰撞过程. 电极直径 从 $10 \mu \mathrm{m}$ 缩小至 $480 \mathrm{~nm}$ 后, 噪音电流的 $\mathrm{RMS}$ 从 $5.9 \mathrm{pA}$ 降至 $2.7 \mathrm{pA}$, 提高了对银纳米颗粒碰撞电极过程中氧化 电流的分辨能力. 因此, 纳米电极可以提高对单体电化 学中微弱电流的检测能力. 目前的单体电化学研究已从 区分纳米颗粒尺寸拓展到单颗粒催化反应、纳米界面光 致电子转移过程、单细胞原位检测等, 纳米电极的应用 将进一步促进对单体性质的深入理解 ${ }^{[13]}$.

\section{4 实验部分}

二茂铁购自 Sigma-Aldrich (St.Louis, MO, USA), 四 丁基六氟磷酸胺购自百灵威, 其他分析纯试剂购自国药 集团. $10 \mu \mathrm{m}$ 铂超微电极购自上海辰华仪器有限公司. 扫描电子显微镜图像由 Zeiss Auriga 场发射电子束/聚焦 离子束双束系统测试. 透射电子显微镜图像由 JEOL 20100F (JEOL Ltd., Japan)测试.

银纳米颗粒通过种子生长法合成：将 $20 \mathrm{~mL} 1 \%$ $(w / V)$ 柠檬酸三钠和 $75 \mathrm{~mL}$ 去离子水于 $250 \mathrm{~mL}$ 圆底烧 瓶中混合. 在磁力搅拌下加热至 $70{ }^{\circ} \mathrm{C}$ 并保持 $15 \mathrm{~min}$. 然后滴加 $1.7 \mathrm{~mL} \mathrm{AgNO}_{3} 1 \%(w / V)$ 溶液, 紧接着加入 2 $\mathrm{mL} 0.1 \%(w / V) \mathrm{NaBH}_{4}$. 混合溶液在 $70{ }^{\circ} \mathrm{C}$ 搅拌 $1 \mathrm{~h}$, 然后 自然冷却至室温, 并将溶液体积补充至 $100 \mathrm{~mL}$. 自此制

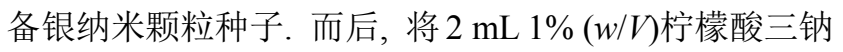
和 $75 \mathrm{~mL}$ 去离子水于 $250 \mathrm{~mL}$ 圆底烧瓶中混合. 在磁力 搅拌下加热至沸腾并保持 $15 \mathrm{~min}$. 然后将溶液剧烈摚 拌, 同时加入 $10 \mathrm{~mL}$ 先前制备的银种溶液, 紧接着加入 $1.7 \mathrm{~mL} \mathrm{1 \% (w/V)} \mathrm{AgNO}_{3}$, 回流 $1 \mathrm{~h}$ 并保持搅拌. 然后两

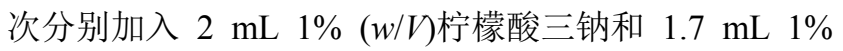
$(w / V) \mathrm{AgNO}_{3}$ 分别回流 $1 \mathrm{~h}$ 并保持搅拌, 最后自然冷却至 室温. 将制备的银纳米颗粒作为种子通过上述方法再生 长两次, 得到直径约为 $60 \mathrm{~nm}$ 的银纳米颗粒.

纳米电极由激光拉制法制备. 首先将长度为 $1 \sim 2$ $\mathrm{cm}$, 直径为 $25 \mu \mathrm{m}$ 的淬火铂丝(Goodfellow, Huntingdon, U.K.) 用导电银胶 (Epoxy Technology, Billerica, MA.
USA)粘附于长为 $5 \mathrm{~cm}$ 左右的铜丝一端, 并于 $120{ }^{\circ} \mathrm{C}$ 固 化 $20 \mathrm{~min}$. 而后将固化的金属丝插入石英玻璃管(O.D.: $1.0 \mathrm{~mm}$, I.D.: $0.5 \mathrm{~mm}$, Sutter, Novato, CA. USA), 并将铂 丝置于玻璃管中心. 而后, 将玻璃管固定于 P-2000 激光 拉制仪卡槽内(Sutter, Novato, CA. USA), 固定 P-2000 激 光拉制仪的两端，同时玻璃管两端连入真空原，抽气 20 $\min$ 保证玻璃管内有较高的真空度. 设置 P-2000 激光拉 制仪的拉制参数为 $(\mathrm{HEAT}=485, \mathrm{FIL}=2, \mathrm{VEL}=60, \mathrm{DEL}$ $=140, \mathrm{PUL}=0)$, 控制加热时间为 $35 \mathrm{~s}$, 冷却时间为 25 $\mathrm{s}$, 重复 5 次. 而后, 移去固定拉制仪两端的装置, 设置 拉制参数为 $(\mathrm{HEAT}=660, \mathrm{FIL}=3, \mathrm{VEL}=60, \mathrm{DEL}=140$, $P U L=225)$, 在真空条件下进行拉制. 最后, 用 EG-401 精密磨针仪(Narishige, Setagaya-ku, Tokyo, Japan)在防静 电措施下进行打磨. 并通过稳态电流法测试其尺寸.

所有电化学信号在 Axon 200B 膜片钳放大器和课 题组自主研发的超微电流检测装置上进行采集. 电流信 号经由 $5 \mathrm{kHz}$ 低通滤波、 $100 \mathrm{kHz}$ 采样频率采集后通过 Clampex 10.4 程序得到(Molecular Devices, Forest City, CA, USA).

\section{References}

[1] Actis, P.; Bentley, C. L.; Edwards, M. A.; Jacobse. L. Chem. Commun. 2016, 52, 13934.

[2] Peng, Y. Y.; Qian, R. C.; Hafez, M. E.; Long, Y. T. ChemElectroChem 2016, DOI: 10. 1002/celc. 201600673.

[3] Li, T.; Liu, Y.; Jiang, Y. N.; Wang, J. H.; Yu, P.; Mao, L. Q. Sci. Sin. Chim. 2016, 46, 1064 (in Chinese). (李婷, 刘洋, 蒋亚楠, 王建华, 于萍, 毛兰群, 中国科学: 化学, 2016, 46, 1064.)

[4] Xiao, X. Y.; Bard, A. J. J. Am. Chem. Soc. 2007, 129, 9610.

[5] Zhou, Y. G.; Rees, N. V.; Compton, R. G. Angew. Chem. Int. Ed. 2011, 50, 4219.

[6] Kim, B. K.; Boika, A.; Kim, J.; Dick, J. E.; Bard, A. J. J. Am. Chem. Soc. 2014, 136, 4849 .

[7] Dunevall, J.; Fathali, H.; Najafinobar, N.; Lovric, J.; Wigstrom, J.; Cans, A. S.; Ewing, A. G. J. Am. Chem. Soc. 2015, 137, 4344.

[8] Cheng, W.; Compton, R. G. Angew. Chem. Int. Ed. 2014, 126, 14148 .

[9] Oja, S. M.; Robinson, D. A.; Vitti, N. J.; Edwards, M. A.; Liu, Y.; White, H. S.; Zhang, B. J. Am. Chem. Soc. 2017, 139, 708.

[10] Ustarroz, J.; Kang, M.; Bullions, E.; Unwin, P. R. Chem. Sci. 2017, 8,1841 .

[11] Ma, W.; Ma, H.; Chen, J. F.; Peng, Y. Y.; Yang, Z. Y.; Wang, H. F.; Ying, Y. L.; Tian, H.; Long, Y. T. Chem. Sci. 2017, 8, 1854.

[12] Ji, T. R.; Liang, Z. W.; Zhu, X. Y.; Shao, Y. H. Chinese J. Anal. Chem. 2010, 12, 1821 (in Chinese). (纪天容, 梁中伟, 朱新宇, 邵 元华, 分析化学, 2010, 12, 1821.)

[13] Ying, Y. L.; Ding, Z. F.; Zhan, D. P.; Long, Y. T. Chem. Sci. 2017, 8, 3338.

[14] Han, L. H.; He, Q. F.; Zhao, X. S.; Cao, Y. Z.; Hu, Z. J.; Yan, Y. D.; Tian, Z. W.; Zhan, D. P. Sci. Sin. Chim. 2017, DOI: 10. 1360/ N032016-00217 (in Chinese). (韩联欢, 何权烽, 赵学森, 曹永智, 胡振江, 间永达, 田昭武, 詹东平, 中国科学: 化学, 2017, DOI: 10. 1360/ N032016-00217.)

[15] Zhou, Y. G.; Rees, N. V.; Compton, R. G. Chem. Commun. 2012, 48, 2510.

[16] Stuart, E. J. E.; Rees, N. V.; Cullen, J. T.; Compton, R. G. Nanoscale 2013, 5, 174.

[17] Kwon, S. J.; Zhou, H.; Fan, F. R. F.; Vorobyev, V.; Zhang, B.; Bard, A. J. Phys. Chem. Chem. Phys. 2011, 13, 5394.

[18] Ahn, H. S.; Bard, A. J. Angew. Chem. Int. Ed. 2015, 127, 13957.

(Cheng, B.; Fan, Y.) 